\title{
Memory-induced acceleration and slowdown of barrier crossing
}

Cite as: J. Chem. Phys. 148, 014903 (2018); https://doi.org/10.1063/1.4998239

Submitted: 29 July 2017. Accepted: 07 December 2017. Published Online: 03 January 2018

Julian Kappler (D), Jan O. Daldrop, Florian N. Brünig, Moritz D. Boehle (D), and Roland R. Netz (D)
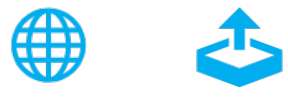

\section{ARTICLES YOU MAY BE INTERESTED IN}

Transition path time distributions

The Journal of Chemical Physics 147, 214103 (2017); https://doi.org/10.1063/1.5000423

Perspective: Maximum caliber is a general variational principle for dynamical systems The Journal of Chemical Physics 148, 010901 (2018); https://doi.org/10.1063/1.5012990

Construction of non-Markovian coarse-grained models employing the Mori-Zwanzig formalism and iterative Boltzmann inversion

The Journal of Chemical Physics 147, 244110 (2017); https://doi.org/10.1063/1.5009041

Where in the world is AIP Publishing?

Find out where we are exhibiting next 


\title{
Memory-induced acceleration and slowdown of barrier crossing
}

\author{
Julian Kappler, Jan O. Daldrop, Florian N. Brünig, Moritz D. Boehle, and Roland R. Netz \\ Freie Universität Berlin, Fachbereich Physik, 14195 Berlin, Germany
}

(Received 29 July 2017; accepted 7 December 2017; published online 3 January 2018)

\begin{abstract}
We study the mean first-passage time $\tau_{\text {MFP }}$ for the barrier crossing of a single massive particle with non-Markovian memory by Langevin simulations in one dimension. In the Markovian limit of short memory time $\tau_{\Gamma}$, the expected Kramers turnover between the overdamped (high-friction) and the inertial (low-friction) limits is recovered. Compared to the Markovian case, we find barrier crossing to be accelerated for intermediate memory time, while for long memory time, barrier crossing is slowed down and $\tau_{\mathrm{MFP}}$ increases with $\tau_{\Gamma}$ as a power law $\tau_{\mathrm{MFP}} \sim \tau_{\Gamma}^{2}$. Both effects are derived from an asymptotic propagator analysis: while barrier crossing acceleration at intermediate memory can be understood as an effective particle mass reduction, slowing down for long memory is caused by the slow kinetics of energy diffusion. A simple and globally accurate heuristic formula for $\tau_{\text {MFP }}$ in terms of all relevant time scales of the system is presented and used to establish a scaling diagram featuring the Markovian overdamped and the Markovian inertial regimes, as well as the non-Markovian intermediate memory time regime where barrier crossing is accelerated and the non-Markovian long memory time regime where barrier crossing is slowed down. Published by AIP Publishing. https://doi.org/10.1063/1.4998239
\end{abstract}

\section{INTRODUCTION}

The rate-determining step of chemical and conformational molecular reactions is typically modeled as the crossing over a single barrier in an effective one-dimensional energy landscape. ${ }^{1-11}$ Solvent reorganization and intra- or intermolecular degrees of freedom that are orthogonal to the reaction coordinate give rise to non-Markovian or memory effects. Many recipes for choosing a good reaction coordinate, i.e., a coordinate for which memory effects are minimal or even absent, have been suggested. ${ }^{12-21}$ The existence of a good coordinate depends on a separation of time scales between orthogonal degrees of freedom and the typical reaction time scale. Only if orthogonal degrees of freedom relax relatively quickly (corresponding to the adiabatic approximation) is a Markovian description possible. ${ }^{22-27}$ In this case, the system kinetics and the transition rate can be characterized by instantaneous friction. In this paper, we consider the general situation where the memory time can be smaller or larger than the other intrinsic time scales of the system and address the question how the barrier crossing time depends on the diffusive, the inertial, and the memory time scales. This encompasses macromolecular reactions when there is no clear time scale separation between the reaction coordinate and the environment and also the case of ill-conditioned reaction coordinates that are coupled to very slow orthogonal degrees of freedom. We are particularly interested in the intermediate scenario when the diffusive, the inertial, and the memory time scales are of the same order, which is relevant for dihedral barrier crossing reactions in peptides and alkanes ${ }^{22}$ as well as for ion-pairing kinetics. ${ }^{20,28}$

Our general viewpoint follows from the fact that the dynamics of a complex multi-dimensional system can be described by the one-dimensional generalized Langevin equation (GLE) $)^{29,30}$

$$
m \ddot{x}(t)=-U^{\prime}(x(t))-\int_{0}^{t} \Gamma\left(t^{\prime}\right) \dot{x}\left(t-t^{\prime}\right) \mathrm{d} t^{\prime}+\eta(t),
$$

where $m$ is the effective mass of the reaction coordinate $x$, $U^{\prime}(x)$ is the derivative of the potential $U(x)$, and $\Gamma(t)$ is the memory kernel that results from integrating out all orthogonal degrees of freedom, which is assumed to couple linearly to the velocity $\dot{x}$. The Gaussian random force $\eta(t)$ has zero mean and in equilibrium obeys the fluctuation-dissipation theorem (FDT)

$$
\left\langle\eta(t) \eta\left(t^{\prime}\right)\right\rangle=k_{\mathrm{B}} T \Gamma\left(t-t^{\prime}\right),
$$

where $k_{\mathrm{B}} T$ is the thermal energy. If the memory kernel $\Gamma(t)$ decays fast compared to the time scale on which $\dot{x}(t)$ varies, one reaches the Markovian limit and Eq. (1) reduces to the ordinary memoryless Langevin equation characterized by the friction coefficient $\gamma=\int_{0}^{\infty} \Gamma(t) \mathrm{d} t$. In this work, we consider a single-exponential memory kernel, ${ }^{31}$

$$
\Gamma(t)=\frac{\gamma}{\tau_{\Gamma}} \exp \left(-\frac{|t|}{\tau_{\Gamma}}\right),
$$

that in addition to $\gamma$ is characterized by the memory time $\tau_{\Gamma}$. This specific form of the memory kernel allows us to vary the friction coefficient $\gamma$ and the memory time $\tau_{\Gamma}$ independently from each other.

We are interested in the mean first-passage time (MFPT) $\tau_{\text {MFP }}$ needed to cross a barrier of height $U_{0}$ in the quartic double well potential defined by

$$
U(x)=U_{0}\left[\left(\frac{x}{L}\right)^{2}-1\right]^{2},
$$


(a)

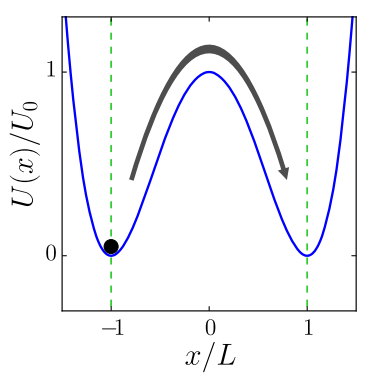

(e)

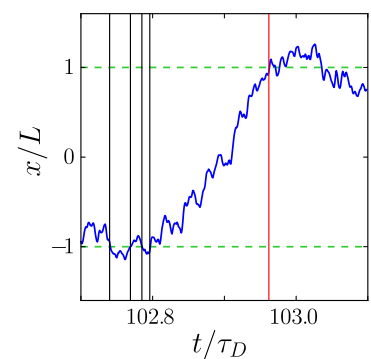

(b)

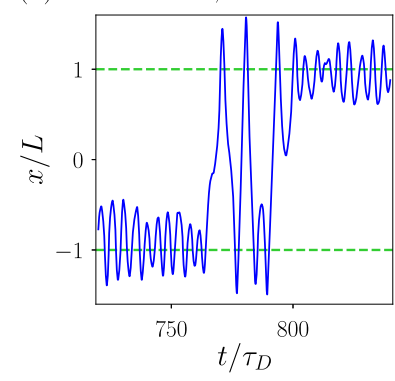

(f)

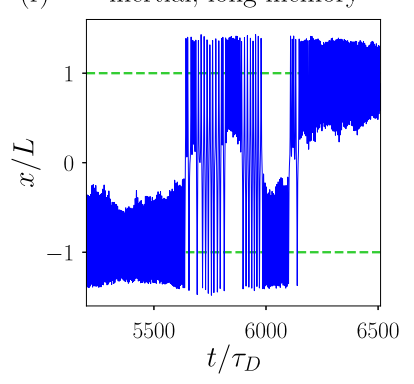

(c)

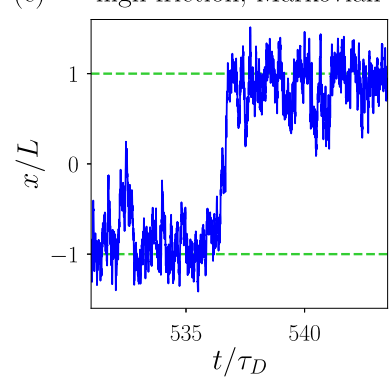

(g) high friction, long memory

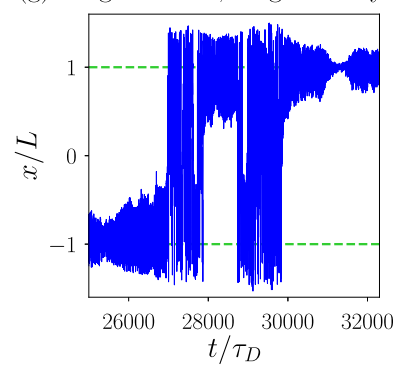

(d)

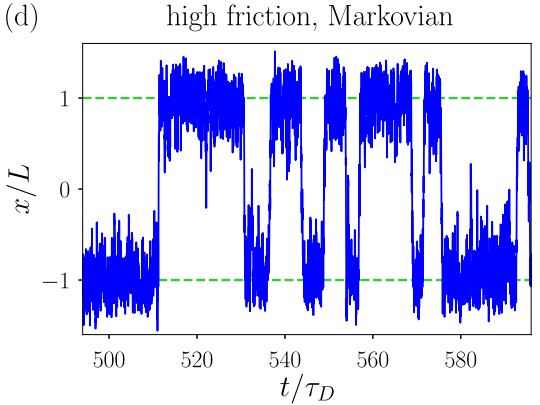

(h)

high friction, long memory

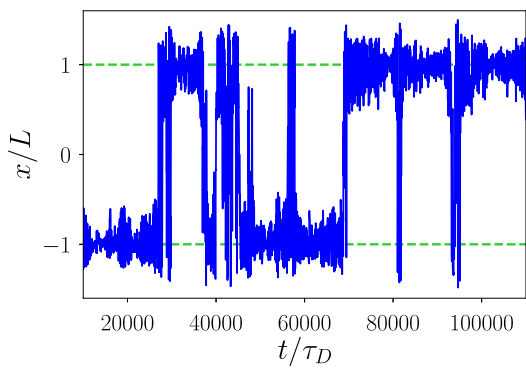

FIG. 1. (a) Illustration of the barrier crossing of a massive particle in the double-well potential $U(x)$ defined in Eq. (4). The mean first-passage time $\tau_{\text {MFP }}$ is defined as the mean time difference between crossing the minimum at $x=-L$ (left vertical dashed line) and reaching the other minimum at $x=L$ (right vertical dashed line) for the first time. [(b)-(d) and (f)-(h)] Typical simulation trajectories that display barrier crossing events for barrier height $U_{0}=3 k_{\mathrm{B}} T$ and (b) low friction $\tau_{m} / \tau_{D}=10$ and short memory $\tau_{\Gamma} / \tau_{D}=0.001$, [(c) and (d)] high friction $\tau_{m} / \tau_{D}=0.001$ and short memory $\tau_{\Gamma} / \tau_{D}=0.001$, (f) low friction $\tau_{m} / \tau_{D}=10$ and long memory $\tau_{\Gamma} / \tau_{D}=10,[(\mathrm{~g})$ and $(\mathrm{h})]$ high friction $\tau_{m} / \tau_{D}=0.001$ and long memory $\tau_{\Gamma} / \tau_{D}=10$. The horizontal green dashed lines indicate the potential minima. (e) Illustration of how first-passage times (FPTs) are obtained from Langevin simulations. The vertical black lines mark crossings of the trajectory with the minimum $x=-L$ (lower green dashed line), and the vertical red line marks the first crossing of the trajectory with the minimum $x=L$ (upper green dashed line). Each vertical black line constitutes a sample for the FPT, obtained via calculating the time difference to the red line, and the MFPT $\tau_{\text {MFP }}$ is obtained by averaging over all FPTs. For the trajectory shown, the parameters $\tau_{m} / \tau_{D}=\tau_{\Gamma} / \tau_{D}=0.001$ and barrier height $U_{0}=3 k_{\mathrm{B}} T$ are used.

see Fig. 1(a) for an illustration. This problem has a long and active history. Early on it was shown that $\tau_{\text {MFP, which equals }}$ the barrier escape time in certain limits, ${ }^{32}$ as will be discussed later, is given by the Arrhenius law $\tau_{\mathrm{MFP}} \sim \exp \left(U_{0} /\left(k_{\mathrm{B}} T\right)\right),{ }^{33}$ but the pre-exponential factor remained unclear. The transition state theory (TST) of Eyring ${ }^{34}$ predicts $\tau_{\text {MFP }}$ in the framework of equilibrium statistical mechanics but does not include the friction coupling to the environment. This gap was filled by Kramers, who for Markovian dynamics, i.e., $\tau_{\Gamma}=0$, derived $\tau_{\text {MFP }}$ for asymptotically small as well as high friction $\gamma .{ }^{35} \mathrm{He}$ found that for fixed mass $m, \tau_{\mathrm{MFP}}$ is minimal for intermediate $\gamma$. Four decades later, the Markovian theory including also the regime for intermediate $\gamma$, known as Kramers turnover, was established by Mel'nikov and Meshkov (MM). ${ }^{36}$ For short memory time $\tau_{\Gamma}$, Grote and Hynes $(\mathrm{GH})$ derived a selfconsistent equation for $\tau_{\text {MFP }}$ in the medium-to-high friction regime, ${ }^{37}$ while Carmeli and Nitzan provided a formula for low friction. ${ }^{38}$ These limiting cases were bridged by empirical expressions. ${ }^{31,39,40}$ Finally, Pollak, Grabert, and Hänggi (PGH) worked out a theory for $\tau_{\mathrm{MFP}}$ for arbitrary memory time $\tau_{\Gamma}$ and arbitrary friction $\gamma .{ }^{41}$

GH and PGH theories can be derived using the equivalence of the GLE, Eq. (1), to a Hamiltonian system where the one-dimensional coordinate $x$ is coupled to harmonic oscillators. ${ }^{42}$ Using a normal-mode approach, the particle barrier escape time can be related to the dynamic energy partitioning between the modes. ${ }^{41}$ In contrast to the GH theory, the PGH theory accounts for the particle history stemming from unsuccessful barrier crossing attempts. ${ }^{41}$ The PGH theory has been recently extended to account for low barriers ${ }^{43,44}$ and has been successfully compared with Langevin simulations in a restricted parameter range. ${ }^{44,45}$ Excellent accounts of the historical development of rate theories ${ }^{46,47}$ and pedagogical introductions to the PGH theory ${ }^{48}$ exist. However, due to the complex mathematical structure of the PGH theory, it is mostly the $\mathrm{GH}$ theory that is currently used for the interpretation of molecular dynamics (MD) simulations. ${ }^{22,49-53}$ The precise limits of accuracy of the various theories and formulations have remained unclear.

In this work, we study the barrier crossing by numerical simulations of the GLE, Eq. (1), in the complete parameter space that encompasses both low and high friction $\gamma$ as well as short and long memory times $\tau_{\Gamma}$. By comparison of our numerical data with existing theories, we confirm that the $\mathrm{GH}$ theory has a very limited range of applicability, ${ }^{31}$ while the PGH theory is virtually exact for all parameters. We find a regime at intermediate memory time where memory accelerates barrier crossing. At long memory time, the MFPT scales as $\tau_{\mathrm{MFP}} \sim \tau_{\Gamma}^{2} e^{U_{0} /\left(k_{\mathrm{B}} T\right)} / \gamma$ and thus increases with $\tau_{\Gamma}$ as a power law, ${ }^{31}$ which shows that memory can modify the barrier crossing behavior at time scales that are much longer than the memory time itself. By an asymptotic dynamic propagator analysis, the barrier crossing acceleration at intermediate $\tau_{\Gamma}$ is explained by effective mass reduction due to unsuccessful barrier crossing attempts, the barrier crossing slowdown at large $\tau_{\Gamma}$ by slow energy diffusion effects. We provide a simple heuristic formula for $\tau_{\text {MFP }}$ that holds globally as a function of all parameters and use it to predict the crossover 
between the Markovian overdamped and inertial regimes and the two non-Markovian regimes where memory effects are relevant and either accelerate or slow down the barrier crossing rate.

\section{SETUP}

To reformulate the problem defined by Eqs. (1)-(4) with a minimal set of parameters, we introduce the time scales

$$
\tau_{D}=\frac{L^{2} \gamma}{k_{\mathrm{B}} T}, \quad \tau_{m}=\frac{m}{\gamma},
$$

where $\tau_{D}$ is the diffusion time linked with the barrier separation $L$ and friction coefficient $\gamma$, and $\tau_{m}$ is the inertial time that characterizes viscous dissipation of particle momentum. With this, we can rewrite the GLE, Eq. (1), as

$$
\tau_{m} \ddot{\tilde{x}}(t)=\frac{4 U_{0}}{k_{\mathrm{B}} T} \frac{\tilde{x}\left(1-\tilde{x}^{2}\right)}{\tau_{D}}-\int_{0}^{t} e^{-t^{\prime} / \tau_{\Gamma}} \dot{\tilde{x}}\left(t-t^{\prime}\right) \frac{\mathrm{d} t^{\prime}}{\tau_{\Gamma}}+\tilde{\eta}(t),
$$

where $\tilde{x}=x / L$ is the dimensionless particle position and $\tilde{\eta}=\eta /(\gamma L)$ is the rescaled random noise that is characterized by the correlator $\left\langle\tilde{\eta}(t) \tilde{\eta}\left(t^{\prime}\right)\right\rangle=\exp \left(-\left|t-t^{\prime}\right| / \tau_{\Gamma}\right) /\left(\tau_{D} \tau_{\Gamma}\right)$. It now transpires that the problem is fully specified by the rescaled potential barrier height $U_{0} /\left(k_{\mathrm{B}} T\right)$ and two dimensionless ratios formed by the characteristic time scales $\tau_{m}, \tau_{D}$, and $\tau_{\Gamma}$.

For numerical simulations of Eq. (6), we eliminate the memory kernel by coupling to an additional fluctuating degree of freedom. ${ }^{31}$ More explicitly, Eq. (6) is equivalent to the coupled system of equations

$$
\begin{aligned}
& \tau_{m} \ddot{\tilde{x}}(t)=\frac{4 U_{0}}{k_{\mathrm{B}} T} \frac{\tilde{x}\left(1-\tilde{x}^{2}\right)}{\tau_{D}}+R(t), \\
& \tau_{\Gamma} \dot{R}(t)=-[R(t)+\dot{\tilde{x}}(t)-\xi(t)],
\end{aligned}
$$

where $\xi$ is white noise with $\langle\xi(t)\rangle=0$ and variance $\left\langle\xi(t) \xi\left(t^{\prime}\right)\right\rangle=2 \delta\left(t-t^{\prime}\right) / \tau_{D}$. The solution to Eq. (8) with initial condition $R(0)=R_{0}$ is $R(t)=R_{0} e^{-t / \tau_{\Gamma}}-\int_{0}^{t} e^{-t^{\prime} / \tau_{\Gamma}}$ $\times\left[\dot{\tilde{x}}\left(t-t^{\prime}\right)-\xi\left(t-t^{\prime}\right)\right] \mathrm{d} t^{\prime} / \tau_{\Gamma}$. Substituting this into Eq. (7), one recovers Eq. (6) with an effective noise given by $\tilde{\eta}(t)=R_{0} e^{-t / \tau_{\Gamma}}+\int_{0}^{t} e^{-t^{\prime} / \tau_{\Gamma}} \xi\left(t-t^{\prime}\right) \mathrm{d} t^{\prime} / \tau_{\Gamma}$. The FDT is fulfilled if $R_{0}$ is drawn from a Gaussian distribution with zero mean and variance $\left\langle R_{0}^{2}\right\rangle=1 /\left(\tau_{\Gamma} \tau_{D}\right)$. In our simulations, we sample the initial position $\tilde{x}(0)$ from the equilibrium distribution within the potential well centered around $\tilde{x}=-1$, using a Gaussian approximation so that $\langle\tilde{x}(0)\rangle=-1$ and $\left\langle(\tilde{x}(0)+1)^{2}\right\rangle$ $=k_{\mathrm{B}} T /\left(U^{\prime \prime}(-L) L^{2}\right)=k_{\mathrm{B}} T /\left(8 U_{0}\right)$. The initial velocity $\dot{\tilde{x}}(0)$ is sampled from a Gaussian distribution with zero mean and variance $\left\langle\dot{\tilde{x}}(0)^{2}\right\rangle=k_{\mathrm{B}} T /\left(L^{2} m\right)=1 /\left(\tau_{m} \tau_{D}\right)$, in accordance with the equipartition theorem. Equations (7) and (8) are integrated numerically using a standard fourth-order Runge-Kutta scheme.

Particle trajectories for overdamped and inertial dynamics with short and long memory times, respectively, are shown in Fig. 1. In Figs. 1(g) and 1(h), it is seen that even in the high friction case, we obtain for long memory time bursts of quickly repeating barrier recrossing events, similar to the inertial Markovian case shown in Fig. 1(b), hinting already at a close analogy between the long-memory and the inertial limits, which we will describe below. ${ }^{31,38,54}$ Most of the results for the MFPT $\tau_{\text {MFP }}$ shown in this paper are extracted from a single long trajectory that in the presence of memory crosses the barrier many times and thus includes the effects of multiple recrossing events. In Appendix A, we discuss alternative estimates of the barrier crossing time based on firstpassage events as well as based on the relaxation dynamics of the particle probability distribution. To obtain $\tau_{\text {MFP }}$, we average over all first passage times, defined as the difference between the time a trajectory crosses the potential minimum at $x=-L$ and the time it reaches the other minimum at $x=L$ for the first time, as illustrated in Fig. 1(e) (to increase sampling efficiency, we also consider the reverse first passage events from $x=L$ to $x=-L$ ). Each of our trajectories includes at least thousand barrier crossing events.

Rate theories typically yield the escape time, $\tau_{\text {esc }}$, which is defined as the inverse of the escape rate at which the fraction of particles initially localized on one side of the barrier decays towards equilibrium. If trajectories cannot recross the barrier once they have reached their target position, corresponding to a single-well scenario which is effected by a suitably positioned absorbing boundary condition, there is no difference between the MFPT $\tau_{\text {MFP }}$ obtained in simulations and $\tau_{\text {esc }}$. If one allows for the recrossing of trajectories, corresponding to the double-well scenario, $\tau_{\text {MFP }}$ and $\tau_{\text {esc }}$ only agree in the high-friction and Markovian limit. For MM and PGH theories, formulas for the escape time in the single-well as well as in the double-well scenarios exist. ${ }^{36,41,48}$ In this paper, we compare our numerically determined MFPTs in the double-well scenario (including barrier recrossing) with the escape times from single-well rate theories (which neglect barrier recrossing). In Appendix A, we present explicit simulation results for doublewell MFPTs and single-well escape times and demonstrate that they are numerically identical. This shows that recrossing events (which are absent in single-well rate theories) contribute negligibly to $\tau_{\mathrm{MFP}}$ and therefore validate our comparison of numerically determined MFPTs in the double-well scenario with escape times from single-well rate theories.

While the results in the main text are based on the quartic potential defined in Eq. (4), in Appendix B, we compare results for quartic and cubic potentials and demonstrate that the differences are insignificant if suitable rescaled variables are used.

\section{COMPARISON OF RATE THEORY RESULTS TO LANGEVIN SIMULATIONS}

Before we display numerical results, we compare in Fig. 2(a) different theoretical predictions for the rescaled MFPT $\tau_{\mathrm{MFP}} / \sqrt{\tau_{D} \tau_{m}}$ for a few different fixed memory times $\tau_{\Gamma}$ and fixed barrier height $U_{0}=3 k_{\mathrm{B}} T$ as a function of the rescaled friction coefficient $\sqrt{\tau_{D} / \tau_{m}} \sim \gamma / \sqrt{m}$. This is the standard way of illustrating the friction-dependent Kramers turnover ${ }^{36,41}$ since the rescaled MFPT $\tau_{\mathrm{MFP}} / \sqrt{\tau_{D} \tau_{m}} \sim \tau_{\mathrm{MFP}} / \sqrt{m}$ does not explicitly depend on $\gamma$. For short memory time $\tau_{\Gamma} / \sqrt{\tau_{D} \tau_{m}}=$ 0.01 , the MM theory (thick gray line) agrees perfectly with the full PGH theory (green line), which is expected since the MM theory is valid in the Markovian limit $\tau_{\Gamma}=0$. For intermediate and long memory times $\tau_{\Gamma} / \sqrt{\tau_{D} \tau_{m}}=1,10$, the PGH theory predicts the MFPT to increase, compared to the Markovian 

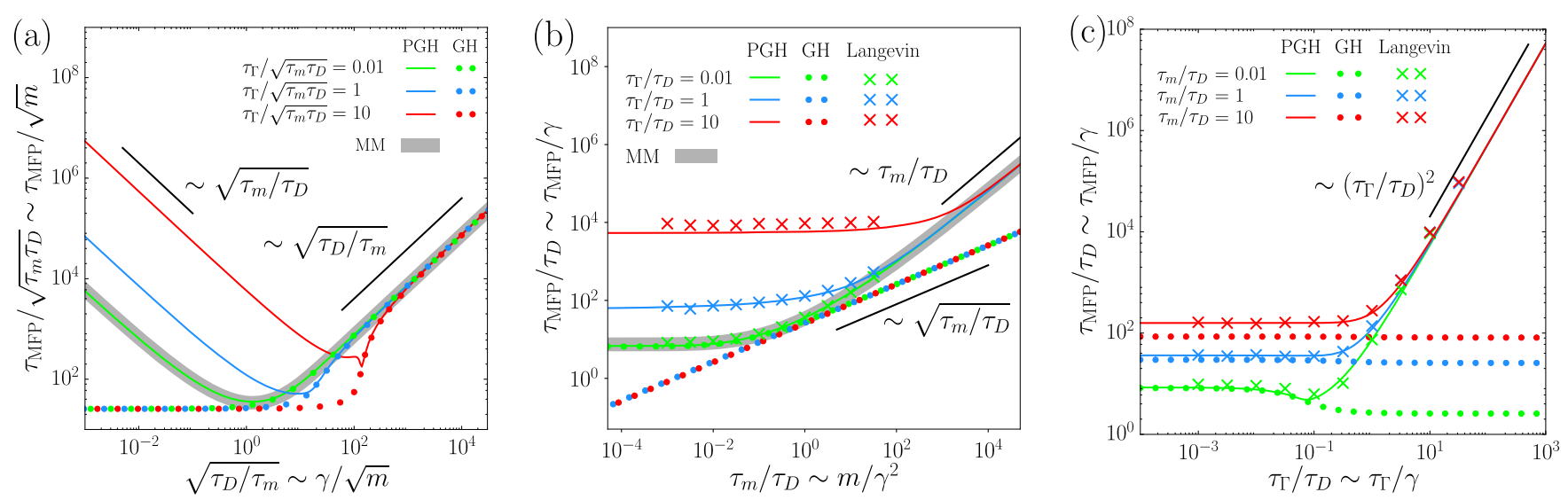

FIG. 2. Simulation results for the mean first-passage time $\tau_{\text {MFP }}$ (crosses) are compared with predictions from the memoryless Mel'nikov-Meshkov (MM) ${ }^{36}$ theory (thick gray line), Grote-Hynes $(\mathrm{GH})^{37}$ theory (dotted lines), and Pollak-Grabert-Hänggi $(\mathrm{PGH})^{41}$ theory (solid colored lines) for fixed barrier height $U_{0}=3 k_{\mathrm{B}} T$. (a) The rescaled MFPT $\tau_{\mathrm{MFP}} / \sqrt{\tau_{m} \tau_{D}} \sim \tau_{\mathrm{MFP}} / \sqrt{m}$ plotted as a function of $\sqrt{\tau_{D} / \tau_{m}} \sim \gamma / \sqrt{m}$ shows the classical Kramers turnover with a minimum in $\tau_{\text {MFP }}$ at an intermediate value of $\gamma / \sqrt{m}$ between the low-friction (to the left) and the high-friction regimes (to the right). The PGH theory converges to the MM theory for short memory, and the GH theory is only valid for high friction. (b) Plot of $\tau_{\mathrm{MFP}} / \tau_{D}$ as a function of $\tau_{m} / \tau_{D}$ for several values of the rescaled memory time $\tau_{\Gamma} / \tau_{D}$. Simulation results agree accurately with the PGH theory. (c) Plot of $\tau_{\mathrm{MFP}} / \tau_{D}$ as a function of $\tau_{\Gamma} / \tau_{D}$ for several values of $\tau_{m} / \tau_{D}$; for long memory, the MFPT scales as $\tau_{\text {MFP }} \sim \tau_{\Gamma}^{2}$. For intermediate values of the rescaled memory time $\tau_{\Gamma} / \tau_{D}$, memory effects in fact accelerate barrier crossing compared to the Markovian (memoryless) case.

limit $\tau_{\Gamma}=0$, in the inertial low-friction limit (to the left), while for high friction, the increase in memory time has no influence on the barrier-crossing time. The power law behavior is independent of the memory time and (for fixed $U_{0} / k_{\mathrm{B}} T$ ) is given by $\tau_{\mathrm{MFP}} \sim m / \gamma$ for low friction and $\tau_{\mathrm{MFP}} \sim \gamma$ for high friction, the standard Kramers scaling. ${ }^{35,36}$ The minimum in the MFPT at intermediate friction is shifted upwards and to larger friction as the memory time increases. The GH theory (dotted lines) clearly breaks down in the inertial regime, which is expected and has been noted before. ${ }^{40}$

To reveal the global scaling structure of the barrier crossing time, it is useful to slightly change the rescaling and to express all times in units of $\tau_{D}$. Figure 2(b) shows $\tau_{\mathrm{MFP}} / \tau_{D}$ as a function of $\tau_{m} / \tau_{D}$ for a few fixed values of the rescaled memory time $\tau_{\Gamma} / \tau_{D}$, crosses denote simulation results, which quantitatively agree with the PGH theory (solid colored lines). The small deviations between simulations and PGH theory for long memory time are discussed in Appendix A. The highfriction regime (to the left) is characterized by a constant rescaled MFPT $\tau_{\mathrm{MFP}} / \tau_{D} \sim$ const. and thus $\tau_{\mathrm{MFP}} \sim \gamma$, while the low-friction regime (to the right) displays a linear scaling $\tau_{\mathrm{MFP}} / \tau_{D} \sim \tau_{m} / \tau_{D}$ and thus $\tau_{\mathrm{MFP}} \sim m / \gamma$ (as indicated by a black bar). Notably, in this presentation, it is seen that the GH theory not only fails in the inertial regime (to the right) but also fails in the diffusive regime for elevated memory times $\tau_{\Gamma} / \tau_{D}$ $=1$ and 10 and reduces to the transition-state theory scaling $\tau_{\mathrm{MFP}} \sim \sqrt{m}$ as indicated by a black bar.

In Fig. 2(c), we present the scaling behavior of the MFPT as a function of the memory time $\tau_{\Gamma}$; for this, we plot $\tau_{\mathrm{MFP}} / \tau_{D}$ versus $\tau_{\Gamma} / \tau_{D}$ for a few different fixed values of $\tau_{m} / \tau_{D}$. Again, simulations (crosses) agree quantitatively with the PGH theory (solid lines) for all parameter values, which demonstrates that the PGH theory is virtually exact both for long and short memory times as well as for low and high friction. The power-law behavior $\tau_{\text {MFP }} \propto \tau_{\Gamma}^{2}$ for long memory times $\tau_{\Gamma} / \tau_{D} \gg 1$, which has been demonstrated before, ${ }^{31}$ is clearly seen both for low and high friction, i.e., for all values of $\tau_{m} / \tau_{D}$. Note that this power law scaling is not captured by the
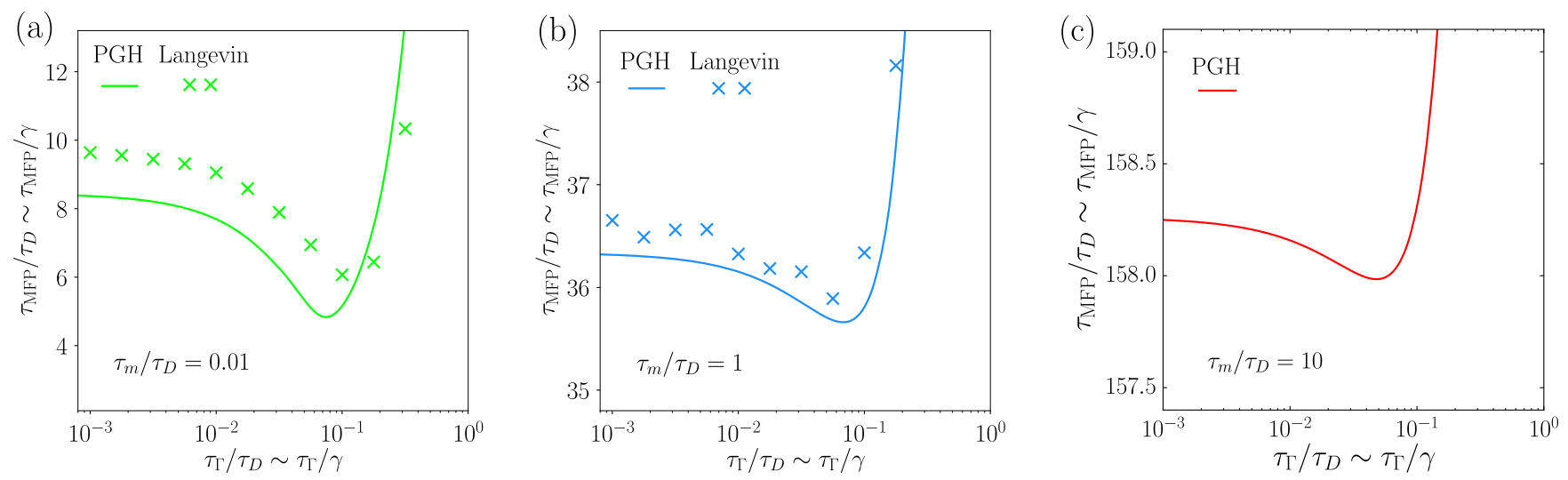

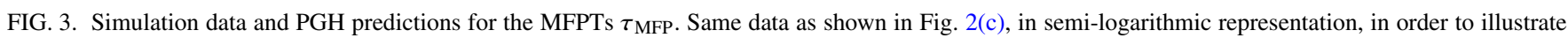
the minimum in $\tau_{\text {MFP }}$ at intermediate memory times $\tau_{\Gamma}$. The numerical results shown in subplots [(a) and (b)] illustrate that the speedup in barrier crossing is also observed in the Langevin simulations. For subplot (c), no numerical data are shown because a speedup could not be observed within statistical error. 

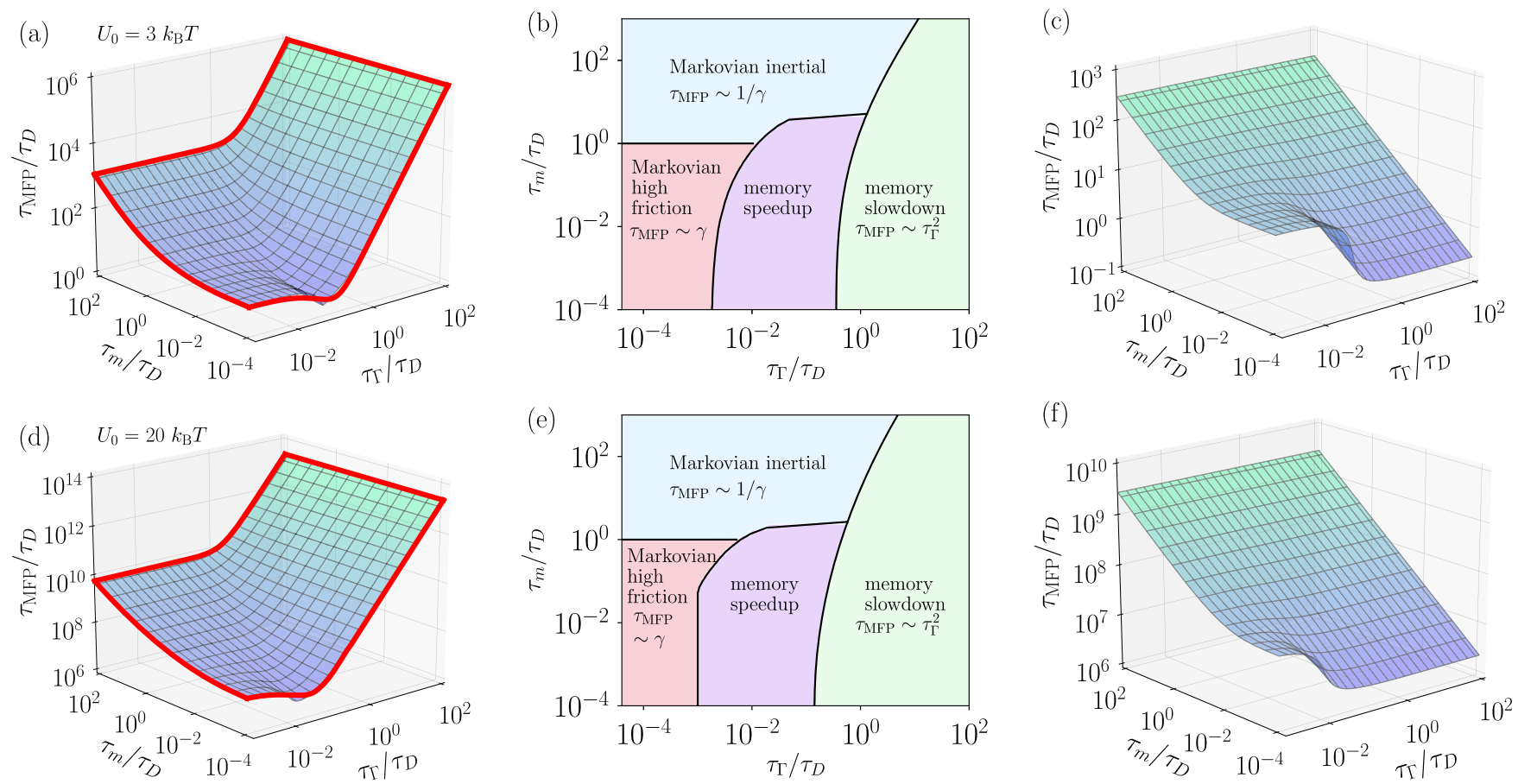

FIG. 4. [(a) and (d)] Global plots of the mean first-passage time $\tau_{\mathrm{MFP}} / \tau_{D}$ as a function of $\tau_{m} / \tau_{D}$ and $\tau_{\Gamma} / \tau_{D}$ according to the PGH theory for barrier heights (a) $U_{0}=3 k_{\mathrm{B}} T$ and (d) $U_{0}=20 k_{\mathrm{B}} T$. The red lines at the boundaries represent the heuristic formula Eq. (9). [(b) and (e)] Scaling diagrams showing the different

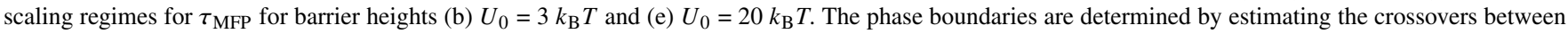
the different scaling forms Eqs. (10)-(13) of the heuristic formula Eq. (9). The memory speedup regime is defined by the parameter range where the heuristic formula (9) yields a value for $\tau_{\mathrm{MFP}} / \tau_{D}$ less than $95 \%$ of the Markovian limit (11). [(c) and (f)] Global plots of the mean first-passage time $\tau_{\mathrm{MFP}} / \tau_{D}$ as a function of $\tau_{m} / \tau_{D}$ and $\tau_{\Gamma} / \tau_{D}$ according to the GH theory for barrier heights (c) $U_{0}=3 k_{\mathrm{B}} T$ and (f) $U_{0}=20 k_{\mathrm{B}} T$, which agrees with the PGH theory only in the short-memory high-friction regime, defined by $\tau_{\Gamma} / \tau_{D} \ll 1$ and $\tau_{m} / \tau_{D} \ll 1$.

GH theory (dotted lines), but rather by the Carmeli-Nitzan energy diffusion formula. ${ }^{31,54}$ Unfortunately, the CarmeliNitzan theory is rarely used when applying rate theories to MD data. In between the asymptotic short- and long-memory regimes, the MFPT shows a minimum, which is most visible for small mass $\tau_{m} / \tau_{D}=0.01$ (shown in green). For the other two friction values shown $\left(\tau_{m} / \tau_{D}=1,10\right)$, a minimum cannot be discerned in the log-log representation, but it is still present. This is more clearly seen in Fig. 3, where we replot the simulation data and the PGH prediction in a semi-logarithmic presentation and obtain quite good agreement between the two. As we will discuss in more detail in Secs. IV and V, intermediate memory time speeds up barrier crossing, while a long memory time invariably slows down reaction rates and in fact gives rise to a power law dependence of $\tau_{\text {MFP }}$ on $\tau_{\Gamma}$.

The three different ways of representing the data in Fig. 2 bring out the three fundamental scaling properties of the MFPT, namely, the scaling $\tau_{\text {MFP }} \sim \gamma$ for high friction, seen in Fig. 2(a) to the right, the scaling $\tau_{\mathrm{MFP}} \sim m / \gamma$ for large mass, seen in Fig. 2(b) to the right, and the scaling $\tau_{\mathrm{MFP}} \sim \tau_{\Gamma}^{2}$ for large memory time, seen in Fig. 2(c) to the right.

To present this scaling behavior of the MFPT in one graph, we show the PGH predictions for $\tau_{\mathrm{MFP}} / \tau_{D}$ in Fig. 4(a) in a triple-logarithmic plot as a function of $\tau_{m} / \tau_{D}$ and $\tau_{\Gamma} / \tau_{D}$ for fixed $U_{0}=3 k_{\mathrm{B}} T . \tau_{\mathrm{MFP}} / \tau_{D}$ shows a plateau in the short-memory high-friction regime for $\tau_{m} / \tau_{D}<1$ and $\tau_{\Gamma} / \tau_{D}<1$. The comparison with Figs. 2(b) and 4(c), where the $\mathrm{GH}$ prediction is shown, demonstrates that this is the only regime where the $\mathrm{GH}$ theory is reliable; this was clear when the GH theory was devised. ${ }^{37}$ For long memory $\tau_{\Gamma} / \tau_{D}$ $\gg \sqrt{\tau_{m} / \tau_{D}}$ (for $\tau_{m} / \tau_{D}>1$ ) or $\tau_{\Gamma} / \tau_{D} \gg 1$ (for $\tau_{m} / \tau_{D}<1$ ), the power law $\tau_{\mathrm{MFP}} / \tau_{D} \sim\left(\tau_{\Gamma} / \tau_{D}\right)^{2}$ emerges in the PGH theory as shown in Fig. 4(a), while the low-friction shortmemory scaling $\tau_{\mathrm{MFP}} / \tau_{D} \sim \tau_{m} / \tau_{D}$ is seen for $\tau_{m} / \tau_{D} \gg 1$ and $\tau_{m} / \tau_{D} \gg\left(\tau_{\Gamma} / \tau_{D}\right)^{2}$.

\section{A SIMPLE HEURISTIC FORMULA FOR THE MEAN FIRST-PASSAGE TIME}

Based on the scaling behavior of $\tau_{\text {MFP }}$ exhibited in Fig. 4(a), we construct the heuristic crossover function

$$
\begin{aligned}
\tau_{\mathrm{MFP}} / \tau_{D}= & e^{U_{0} /\left(k_{\mathrm{B}} T\right)}\left[\frac{\left(U_{0} /\left(k_{\mathrm{B}} T\right)\right)^{-1}}{1+10 U_{0} /\left(k_{\mathrm{B}} T\right) \tau_{\Gamma} / \tau_{D}}+\left(\frac{U_{0}}{k_{\mathrm{B}} T}\right)^{-1} \frac{\tau_{m}}{\tau_{D}}\right. \\
& \left.+2 \sqrt{\left(\frac{U_{0}}{k_{\mathrm{B}} T}\right)^{-1} \frac{\tau_{m}}{\tau_{D}}}+e\left(\frac{\tau_{\Gamma}}{\tau_{D}}\right)^{2}\right]
\end{aligned}
$$

where $e \approx 2.72$ is Euler's number. Equation (9) is plotted in Figs. 4(a) and 4(d) as a red thick line at the boundaries and reproduces the PGH theory very accurately.

In Fig. 5, we compare simulation results for $\tau_{\mathrm{MFP}}$ (crosses) with the PGH theory (solid lines) and our heuristic formula Eq. (9) (dotted lines) as a function of the barrier height $U_{0}$. We compare two representative scenarios, one for high friction $\tau_{m} / \tau_{D}=0.1$ and long memory $\tau_{\Gamma} / \tau_{D}=10$ (in green) and the other for low friction $\tau_{m} / \tau_{D}=10$ and short memory $\tau_{\Gamma} / \tau_{D}=0.1$ (in blue). The heuristic formula Eq. (9) and PGH 


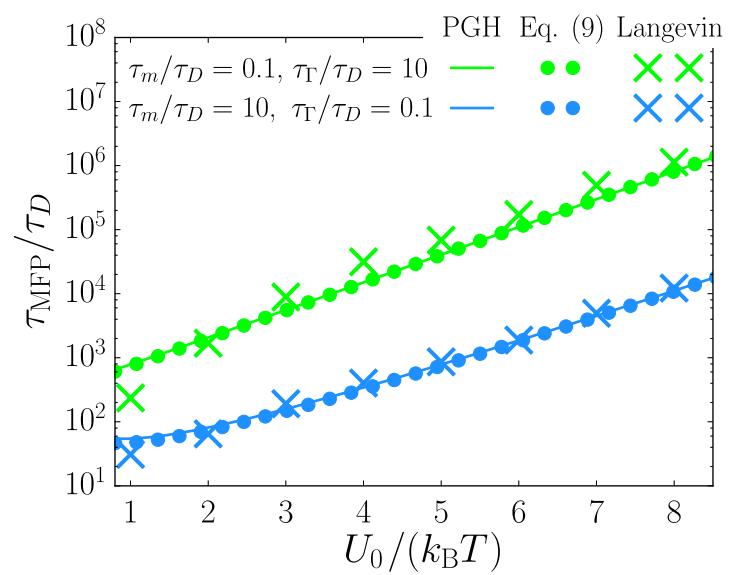

FIG. 5. Mean first-passage time $\tau_{\mathrm{MFP}} / \tau_{D}$ as a function of barrier height $U_{0}$ for two different parameter combinations of $\tau_{m} / \tau_{D}$ and $\tau_{\Gamma} / \tau_{D}$. Crosses denote simulation results, solid lines cenote predictions from the PGH theory, and dotted lines represent the heuristic formula Eq. (9).

theory agree closely with each other and also with the simulation results for not too low barrier heights. It transpires that the low-barrier corrections to the PGH theory ${ }^{43-45}$ are not significant for barrier heights $U_{0} \approx 3 k_{\mathrm{B}} T$. In Appendix C, we present a quantitative comparison of Eq. (9) to the PGH theory predictions and find that throughout the parameter range

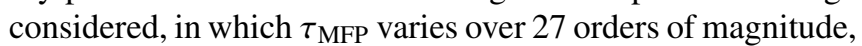
relative deviations between our heuristic formula and the full PGH theory are small. Thus, over many orders of magnitude in the parameters $\tau_{m} / \tau_{D}, \tau_{\Gamma} / \tau_{D}$, and $U_{0}$ and especially in the asymptotic regimes, our formula (9) is a simple and accurate means for calculating $\tau_{\text {MFP. }}$.

Equation (9) allows us to establish a global scaling diagram. While in the long memory limit $\tau_{\Gamma} / \tau_{D} \gg 1$, Eq. (9) reduces to

$$
\tau_{\mathrm{MFP}} / \tau_{D} \approx e^{U_{0} /\left(k_{\mathrm{B}} T\right)+1}\left(\frac{\tau_{\Gamma}}{\tau_{D}}\right)^{2}
$$

in the Markovian limit $\tau_{\Gamma} / \tau_{D} \ll 1$, one obtains

$$
\tau_{\mathrm{MFP}} / \tau_{D} \approx e^{U_{0} /\left(k_{\mathrm{B}} T\right)}\left(\frac{U_{0}}{k_{\mathrm{B}} T}\right)^{-1}\left[1+\frac{\tau_{m}}{\tau_{D}}+2 \cdot \sqrt{\frac{U_{0}}{k_{\mathrm{B}} T} \frac{\tau_{m}}{\tau_{D}}}\right] .
$$

The boundary between the Markovian and the non-Markovian regimes follows by equating Eqs. (10) and (11) and constitutes the left boundary of the memory slowdown regime, shown in green in the scaling diagram for barrier height $U_{0}=3 k_{\mathrm{B}} T$ in Fig. 4(b). The asymptotic boundaries are given by $\left(\tau_{\Gamma} / \tau_{D}\right)^{2}=k_{\mathrm{B}} T / U_{0}$ for high friction $\tau_{m} / \tau_{D} \ll 1$ and $\left(\tau_{\Gamma} / \tau_{D}\right)^{2}=k_{\mathrm{B}} T / U_{0} \cdot \tau_{m} / \tau_{D}$ for low friction $\tau_{m} / \tau_{D} \gg 1$. Similarly, the crossover from the high-friction Markovian limit $\left(\tau_{m} / \tau_{D} \ll 1\right)$ of Eq. $(11)$,

$$
\tau_{\mathrm{MFP}} / \tau_{D} \approx e^{U_{0} /\left(k_{\mathrm{B}} T\right)}\left(\frac{U_{0}}{k_{\mathrm{B}} T}\right)^{-1},
$$

to the low-friction Markovian limit $\left(\tau_{m} / \tau_{D} \gg 1\right)$ of

$$
\tau_{\mathrm{MFP}} / \tau_{D} \approx e^{U_{0} /\left(k_{\mathrm{B}} T\right)}\left(\frac{U_{0}}{k_{\mathrm{B}} T}\right)^{-1} \frac{\tau_{m}}{\tau_{D}}
$$

occurs at $\tau_{m} / \tau_{D}=1$ and separates the Markovian low-friction (inertial) from the Markovian high-friction (overdamped)

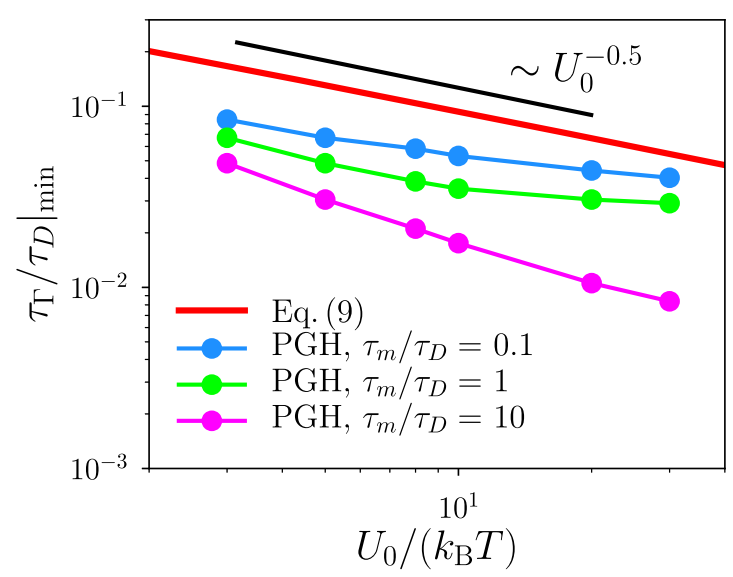

FIG. 6. Memory time which minimizes $\tau_{\mathrm{MFP}} / \tau_{D}$ for given $\tau_{m} / \tau_{D}$, as predicted by Eq. (9) (red solid line) and the PGH theory (data points), as a function of $U_{0}$. Note that according to Eq. (9), $\tau_{\Gamma} /\left.\tau_{D}\right|_{\min }$ does not depend on $\tau_{m} / \tau_{D}$. The power law $\tau_{\Gamma} /\left.\tau_{D}\right|_{\min } \sim 1 / \sqrt{U_{0}}$, denoted by a black bar, approximates well the result from Eq. (9).

regimes in Fig. 4(b). We define the regime within which memory accelerates barrier crossing as the parameter range where $\tau_{\mathrm{MFP}} / \tau_{D}$ according to the heuristic formula Eq. (9) is smaller by $5 \%$ compared to the Markovian limit (11). This defines the memory speedup regime, which in Fig. 4(b) is shown in purple and is obtained for intermediate memory time and not too large mass. Note that while a slight speedup is obtained even for large mass $\tau_{m} / \tau_{D}$, the relative speedup goes to zero as $\tau_{m} / \tau_{D} \gg 1$, as is clearly seen in Fig. 3 .

In Fig. 4(e), we show the scaling diagram for $U_{0}=20 k_{\mathrm{B}} T$. Compared to the result for $U_{0}=3 k_{\mathrm{B}} T$ in (b), the boundary of the memory slowdown regime (shown in green) shifts to slightly smaller values of $\tau_{\Gamma} / \tau_{D}$. Otherwise, the scaling diagrams for $U_{0}=3 k_{\mathrm{B}} T$ and $U_{0}=20 k_{\mathrm{B}} T$ are very similar, which shows that the general scaling structure of the MFPT is robust with respect to variations of the barrier energy $U_{0}$. The comparison of the PGH theory and our heuristic formula for $U_{0}=20 k_{\mathrm{B}} T$ in Fig. 4(d) shows that the comparison is good also for high barriers.

In Fig. 6, we show the memory time $\tau_{\Gamma} /\left.\tau_{D}\right|_{\min }$ at which, for given inertial time scale $\tau_{m} / \tau_{D}$ and barrier height $U_{0}$, the MFPT $\tau_{\mathrm{MFP}} / \tau_{D}$ is minimal. We compare results from the heuristic formula Eq. (9) with predictions of the PGH theory. According to Eq. (9), $\tau_{\Gamma} /\left.\tau_{D}\right|_{\min }$ is independent of $\tau_{m} / \tau_{D}$ and decays as a power law $\tau_{\Gamma} /\left.\tau_{D}\right|_{\text {min }} \sim 1 / \sqrt{U_{0}}$ with $U_{0}$. While $\tau_{\Gamma} / \tau_{D} l_{\min }$ as predicted by the PGH theory does slightly depend on $\tau_{m} / \tau_{D}$ and is smaller than the value obtained from the heuristic formula, the general agreement is quite good.

\section{PROPAGATOR ANALYSIS}

The scaling $\tau_{\mathrm{MFP}} \sim \tau_{\Gamma}^{2}$ for long memory time has been observed before and can be derived from the energy diffusion limit of the barrier crossing rate. ${ }^{31}$ Alternatively, it can be obtained by an asymptotic analysis of the propagator $C(t) \equiv\langle x(t) x(0)\rangle$ that describes the particle motion within the potential well. This approach has the advantage that one can also derive the barrier-crossing speedup at intermediate memory time in a straightforward fashion. 
We consider the GLE (1) in a harmonic potential $U(x) \simeq K x^{2} / 2$ and for times $t \gg \tau_{\Gamma}$ so that we can replace the upper limit in the memory integral by infinity. Fourier transforming Eq. (1) and solving for $\tilde{x}(\omega)$ yields

$$
\tilde{x}(\omega)=\frac{\tilde{\eta}(\omega)}{K-m \omega^{2}+i \omega \tilde{\Gamma}_{+}(\omega)} \equiv \tilde{Q}(\omega) \tilde{\eta}(\omega),
$$

where the half-sided Fourier transform $\tilde{\Gamma}_{+}$of the memory kernel $\Gamma(t)$ is given by

$$
\tilde{\Gamma}_{+}(\omega)=\int_{0}^{\infty} \mathrm{d} t e^{-i \omega t} \Gamma(t)=\frac{\gamma}{1+i \omega \tau_{\Gamma}},
$$

while the Fourier transform of the full memory kernel is

$$
\tilde{\Gamma}(\omega)=\tilde{\Gamma}_{+}(\omega)+\tilde{\Gamma}_{+}(-\omega)=\frac{2 \gamma}{1+\omega^{2} \tau_{\Gamma}^{2}} .
$$

Using Eq. (14), we calculate $\langle x(t) x(0)\rangle$ as

$$
\begin{aligned}
C(t) & \equiv\langle x(t) x(0)\rangle \\
& =\int \frac{\mathrm{d} \omega}{2 \pi} e^{i \omega t} \int \frac{\mathrm{d} \omega^{\prime}}{2 \pi}\left\langle\tilde{x}(\omega) \tilde{x}\left(\omega^{\prime}\right)\right\rangle \\
& =\int \frac{\mathrm{d} \omega}{2 \pi} e^{i \omega t} \int \frac{\mathrm{d} \omega^{\prime}}{2 \pi} \tilde{Q}(\omega) \tilde{Q}\left(\omega^{\prime}\right)\left\langle\tilde{\eta}(\omega) \tilde{\eta}\left(\omega^{\prime}\right)\right\rangle \\
& =k_{\mathrm{B}} T \int \frac{\mathrm{d} \omega}{2 \pi} e^{i \omega t} \int \frac{\mathrm{d} \omega^{\prime}}{2 \pi} 2 \pi \delta\left(\omega+\omega^{\prime}\right) \tilde{\Gamma}(\omega) \tilde{Q}(\omega) \tilde{Q}\left(\omega^{\prime}\right) \\
& =k_{\mathrm{B}} T \int \frac{\mathrm{d} \omega}{2 \pi} e^{i \omega t} \tilde{\Gamma}(\omega) \tilde{Q}(\omega) \tilde{Q}(-\omega),
\end{aligned}
$$

where we used that the Fourier transform of the generalized FDT, Eq. (2), is $\left\langle\tilde{\eta}(\omega) \tilde{\eta}\left(\omega^{\prime}\right)\right\rangle=k_{\mathrm{B}} T 2 \pi \delta\left(\omega+\omega^{\prime}\right) \tilde{\Gamma}(\omega)$. Thus the propagator, i.e., the Fourier transform of $C(t)$, is finally given by

$$
\begin{aligned}
\tilde{C}(\omega) /\left(k_{\mathrm{B}} T\right)= & \tilde{\Gamma}(\omega) \tilde{Q}(\omega) \tilde{Q}(-\omega) \\
= & \frac{2 \gamma}{1+\omega^{2} \tau_{\Gamma}^{2}} \frac{1}{K-m \omega^{2}+i \omega \tilde{\Gamma}_{+}(\omega)} \\
& \times \frac{1}{K-m \omega^{2}-i \omega \tilde{\Gamma}_{+}(-\omega)} \\
= & 2 \gamma\left[\left(K-m \omega^{2}\right)^{2}+\omega^{2} \gamma^{2}+2 \omega^{2} \gamma \tau_{\Gamma}\left(K-m \omega^{2}\right)\right. \\
& \left.+\omega^{2} \tau_{\Gamma}^{2}\left(K-m \omega^{2}\right)^{2}\right]^{-1}
\end{aligned}
$$

For vanishing memory time, $\tau_{\Gamma}=0$, we obtain the standard damped harmonic oscillator result,

$$
\tilde{C}(\omega) /\left(k_{\mathrm{B}} T\right)=2 \gamma\left[\left(K-m \omega^{2}\right)^{2}+\omega^{2} \gamma^{2}\right]^{-1} .
$$

We now investigate the properties of Eq. (24) for small and large memory times $\tau_{\Gamma}$.

For small $\tau_{\Gamma}$, we rewrite Eq. (24) as

$$
\begin{aligned}
\tilde{C}(\omega) /\left(k_{\mathrm{B}} T\right)= & 2 \gamma\left[\left(K-\omega^{2} m\left(1-\tau_{\Gamma} / \tau_{m}\right)\right)^{2}+\omega^{2} \gamma^{2}\right. \\
& +\omega^{2} \tau_{\Gamma}^{2}\left(K-m \omega^{2}-\omega^{2} \gamma^{2}\right]^{-1} \\
= & 2 \gamma\left[\left(K-\omega^{2} m\left(1-\tau_{\Gamma} / \tau_{m}\right)\right)^{2}+\omega^{2} \gamma^{2}\right. \\
& \left.+\mathcal{O}\left(\omega^{2} \tau_{\Gamma}^{2}\right)\right]^{-1} .
\end{aligned}
$$

Neglecting the $\mathcal{O}\left(\omega^{2} \tau_{\Gamma}^{2}\right)$ terms in Eq. (27), we see that Eq. (27) reduces to the memoryless propagator Eq. (25) with an effective mass

$$
m_{\mathrm{eff}}=m\left(1-\tau_{\Gamma} / \tau_{m}\right)
$$

which is smaller than the bare mass $m$. Thus, short but finite memory effectively leads to a reduction of the mass, which according to the Kramers theory is associated with a speedup of the dynamics. This is indeed what we observe in both the Langevin simulations and the PGH theory predictions; see Figs. 2(c) and 3. Note that Eq. (28) was derived from the linearized GLE. To arrive at Eq. (28) in the general nonlinear case, one can alternatively perform a gradient expansion of the memory integral in the GLE, Eq. (1). If the memory kernel $\Gamma$ decays quickly compared to the time scale on which $\dot{x}$ varies, the Taylor expansion of $\dot{x}\left(t-t^{\prime}\right)$ around $t$ and using the exponential memory kernel, Eq. (3), yields

$\int_{0}^{\infty} \Gamma\left(t^{\prime}\right) \dot{x}\left(t-t^{\prime}\right) \mathrm{d} t^{\prime} \approx \int_{0}^{\infty} \Gamma\left(t^{\prime}\right) \mathrm{d} t^{\prime} \dot{x}(t)-\int_{0}^{\infty} t^{\prime} \Gamma\left(t^{\prime}\right) \mathrm{d} t^{\prime} \ddot{x}(t)$

$$
=\gamma \dot{x}(t)-m \frac{\tau_{\Gamma}}{\tau_{m}} \ddot{x}(t) .
$$

Replacing the memory integral in Eq. (1) by this expression, one obtains the ordinary Langevin equation with friction coefficient $\gamma$ and the effective mass $m_{\text {eff }}$ given by Eq. (28), in agreement with our propagator analysis.

For large $\tau_{\Gamma}$, we rewrite Eq. (24) as

$$
\begin{aligned}
\tilde{C}(\omega) /\left(k_{\mathrm{B}} T\right)= & 2 \frac{\gamma}{\omega^{2} \tau_{\Gamma}^{2}}\left[\left(K-m \omega^{2}\right)^{2}+\frac{1}{\omega^{2} \tau_{\Gamma}^{2}}\left(\left(K-m \omega^{2}\right)\right.\right. \\
& \left.\left.+\omega^{2} \gamma^{2}+2 \omega^{2} \gamma \tau_{\Gamma}\left(K-m \omega^{2}\right)\right)\right]^{-1} \\
= & 2 \frac{\gamma}{\omega^{2} \tau_{\Gamma}^{2}}\left[\left(K-m \omega^{2}\right)^{2}+\mathcal{O}\left(\left(\omega \tau_{\Gamma}\right)^{-1}\right)\right]^{-1} \\
= & 2 \gamma_{\mathrm{eff}}\left[\left(K-m \omega^{2}\right)^{2}+\mathcal{O}\left(\left(\omega \tau_{\Gamma}\right)^{-1}\right)\right]^{-1}
\end{aligned}
$$

To leading order in $\tau_{\Gamma}^{-1}$, this is the low friction limit $\tau_{m} \omega \gg 1$ of the memoryless propagator Eq. (25) with a frequency dependent effective friction coefficient $\gamma_{\mathrm{eff}}=\gamma /\left(\omega^{2} \tau_{\Gamma}^{2}\right)$. Indeed, even though the friction coefficient is very high, the longmemory trajectories shown in Figs. 1(g) and 1(h) look inertial, in the sense that once a barrier crossing event takes place, there is a cascade of recrossings, reminiscent of the low-friction barrier crossing, as shown in Fig. 1(b). For low effective friction, the propagator Eq. (25) is dominated by the pole at $\omega_{*}^{2}=K / m$ so that the effective friction asymptotically equals $\gamma_{\text {eff }}=\gamma /\left(\omega_{*}^{2} \tau_{\Gamma}^{2}\right)=\gamma m /\left(K \tau_{\Gamma}^{2}\right)$. Using the Kramers scaling for $\tau_{\text {MFP }}$ for low friction and replacing $\gamma$ by the effective friction coefficient $\gamma_{\text {eff }}$, we finally obtain

$$
\begin{aligned}
\tau_{\mathrm{MFP}} & \sim e^{U_{0} /\left(k_{\mathrm{B}} T\right)} m k_{\mathrm{B}} T /\left(\gamma_{\mathrm{eff}} U_{0}\right) \\
& =e^{U_{0} /\left(k_{\mathrm{B}} T\right)} \tau_{\Gamma}^{2} K k_{\mathrm{B}} T /\left(\gamma U_{0}\right) \\
& =8 e^{U_{0} /\left(k_{\mathrm{B}} T\right)} \tau_{\Gamma}^{2} k_{\mathrm{B}} T /\left(\gamma L^{2}\right) \\
& \sim e^{U_{0} /\left(k_{\mathrm{B}} T\right)} \tau_{\Gamma}^{2} / \tau_{D},
\end{aligned}
$$

where we used $K=8 U_{0} / L^{2}$, appropriate for the quartic potential Eq. (4). This is precisely the scaling behavior obtained for large memory times $\tau_{\Gamma}$ both for large and small bare friction $\gamma$ in Fig. 2(c) and which is also recovered by the heuristic formula Eq. (9).

This scaling result is noteworthy for several reasons. First, it demonstrates that the non-Markovian limit, corresponding 
to memory times $\tau_{\Gamma}$ much larger than the diffusion and inertial times $\tau_{D}$ and $\tau_{m}$, is characterized by a very simple and universal scaling of the barrier crossing time $\tau_{\text {MFP. Second, }}$ orthogonal degrees of freedom, which are at the core of nonMarkovian effects, ${ }^{29,30}$ modify $\tau_{\text {MFP }}$ in a crucial but intuitive manner. To see this, we assume that the non-Markovian effects are caused by a single orthogonal degree of freedom subject to an energy barrier of height $W_{0}$ so that the memory time can be written as $\tau_{\Gamma} \sim \gamma e^{W_{0} /\left(k_{\mathrm{B}} T\right)}$ to leading order. Combining this with the scaling $\tau_{\mathrm{MFP}} \sim e^{U_{0} /\left(k_{\mathrm{B}} T\right)} \tau_{\Gamma}^{2} / \gamma$, we obtain $\tau_{\mathrm{MFP}} \sim \gamma e^{\left(U_{0}+2 W_{0}\right) /\left(k_{\mathrm{B}} T\right)}$. This shows that in the long-memory limit, the orthogonal barrier height $W_{0}$ is even more relevant than the barrier height $U_{0}$ associated with the reaction coordinate, which presumably reflects multiple recrossing events over the orthogonal barrier.

A simple picture allows us to rationalize the observed acceleration of the barrier crossing at intermediate memory time in an intuitive manner. Consider a particle that has just returned from an unsuccessful barrier-crossing attempt and is located at the potential minimum. For a memory time that is of the order of the time it took the particle to move down from the barrier, the net effect of friction will be to accelerate the particle up the barrier again, so this is a simple way of understanding the effective mass reduction expressed by Eq. (28). For much longer memory times, the memory kernel will average over many previous barrier crossing attempts which cancel out on average and thus lead to a reduced effective friction. This explains why the particle trajectories in Figs. 1(g) and 1(h) look inertial and why the kernel derived in the long-memory limit, Eq. (33), has a similar pole structure as the memoryless kernel Eq. (25) for vanishing friction.

\section{CONCLUSIONS}

In summary, we compare several rate theories (MM, GH, PGH) for the barrier-crossing time with explicit Langevin simulation results and determine the range of applicability of the various theoretical predictions. We confirm that the commonly used GH theory is only applicable in the double limit of short memory and high-friction (overdamped) dynamics and that the PGH theory is very accurate in predicting MFPTs in the entire parameter space. From asymptotic analysis of the propagator, we derive that there is a regime at intermediate memory times where memory leads to a decrease of the MFPT $\tau_{\text {MFP }}$ and a distinct regime at long memory times where memory effects slow down barrier crossing and the asymptotic power law $\tau_{\mathrm{MFP}} \sim \tau_{\Gamma}^{2}$ is obtained. As an easy-to-implement alternative to the PGH formula, we provide the heuristic formula (9), show that it accurately predicts the MFPT over many orders of magnitude in the parameters $\tau_{m} / \tau_{D}, \tau_{\Gamma} / \tau_{D}, U_{0}$, and use it to establish the scaling diagram featuring the Markovian highfriction and inertial regimes as well as the non-Markovian long memory regime. In particular, we identify a regime at intermediate memory times where finite memory reduces the barrier crossing time.

The heuristic formula we provide is a convenient tool for the calculation of rates from known memory times, or, conversely, for the estimation of memory times from barrier crossing times measured in MD simulations or experiments. Based on the heuristic formula, we estimate the memory time which maximizes the barrier crossing rate; this might be useful for the design of systems with optimal barrier flux by tuning memory time and barrier height. More generally, our analysis allows us to quickly determine whether memory effects are relevant or not, as summarized in Figs. 4(b) and 4(e). The location in the scaling diagrams where the memory time, the diffusion time, and the inertial time are all of the same order, i.e., $\tau_{\Gamma} / \tau_{D} \sim \tau_{m} / \tau_{D} \sim 1$, is experimentally relevant for dihedral barrier crossing phenomena ${ }^{22,55}$ and ion-pair kinetics in water ${ }^{20,28}$ since in both cases the diffusion time, the memory time, and the inertial time are all of the order of around 1 ps. The scaling diagrams, Figs. 4(b) and 4(e), show that in this area the three scaling regimes corresponding to the memory slowdown, memory speedup, and inertial regimes touch. Since in this regime, neither the Markovian models nor the GH theory, which are easy to handle, is reliable, our heuristic formula will be useful as a convenient and accurate tool to estimate reaction rates for such systems.

\section{ACKNOWLEDGMENTS}

We thank the Deutsche Forschungsgemeinschaft (DFG) for support via Grant No. SFB 1114 in Project No. B02 "Polymer dynamic theories, Markov state models and protein folding free energy landscapes."

\section{APPENDIX A: MEAN FIRST-PASSAGE TIMES VERSUS ESCAPE TIMES AND DOUBLE-WELL VERSUS SINGLE-WELL SCENARIOS}

In the main text, we perform Langevin simulations in a quartic double well defined in Eq. (4) and extract MFPTs from a single long simulation trajectory that includes multiple recrossing events. We compare these simulation results to the PGH theory using an explicit formula for the escape time of particles in a single potential well, which obviously does not allow for barrier recrossing. ${ }^{41}$ The PGH theory has been adapted to calculate the escape time also in a double well, ${ }^{41,47,48}$ which allows for recrossing events, using a method introduced by Mel'nikov and Meshkov, ${ }^{36}$ so the obvious question arises why we do not compare our MFPT simulation results with the PGH theory for a double well.

In this appendix, we first define and discuss the differences between escape and mean first-passage times and between single-well and double-well scenarios. After that we explain why we compare our MFPTs obtained from simulations of a particle in a double-well potential with the single-well PGH formula for the escape time. We finally compare the escape time predictions of the single- and double-well PGH theories with the MFPTs and escape times extracted from Langevin simulations of single- and double wells.

In the single-well PGH theory, ${ }^{41}$ the escape time, $\tau_{\text {esc }}$, defined as the inverse of the escape rate, is obtained from the probability flux out of a potential well with an absorbing boundary condition located to the right of the barrier top so that the particle has negligible probability to recross, assuming a steady-state probability distribution within the well. 
The escape time $\tau_{\text {esc }}$ is defined by the rate equation ${ }^{36,41}$

$$
\tau_{\text {esc }} \dot{P}(t)=-P(t)
$$

for the probability $P(t)$ to find the particle in the well. Starting from the initial condition $P(0)=1$, the solution of Eq. (A1) is

$$
P(t)=e^{-t / \tau_{\mathrm{esc}}} .
$$

The extension of the PGH theory to the double-well scenario only makes a difference in the limits of large mass or long memory and includes barrier recrossing events. ${ }^{36}$ In such recrossing events, a particle recrosses the barrier several times after having reached its target position, which is caused by slow energy diffusion. According to our use of the term, recrossing is a characteristic property of trajectories in the inertial or long memory limits, as shown in Figs. 1(b) and 1(f)-1(h). It should not be mixed up with Markovian barrier recrossing which occurs in the high-friction short-memory limit and consists of the trivial scenario of a particle returning to the starting well after having reached the barrier top. Recrossing events induce a probability influx from the target well so that the escape rate from the starting well is decreased as compared to a single well. In a symmetric double well, the escape time $\tau_{\text {esc }}$ follows from the coupled rate equations for the probabilities to find the particle in the starting well $P_{s}$ and the target well $P_{t}^{36,41}$

$$
\begin{aligned}
& \tau_{\text {esc }} \dot{P}_{s}(t)=-P_{s}(t)+P_{t}(t), \\
& \tau_{\text {esc }} \dot{P}_{t}(t)=P_{s}(t)-P_{t}(t) .
\end{aligned}
$$

If the particle resides in the starting well at time $t=0$, we have $P_{s}(0)=1$ and $P_{t}(0)=0$ so that the solution reads

$$
P_{s}(t)=\frac{1}{2}\left(1+e^{-2 t / \tau_{\mathrm{esc}}}\right) .
$$

Probabilities to be in one well can easily be estimated numerically so that Eqs. (A2) and (A5) can be used to obtain the escape time $\tau_{\text {esc }}$ also from simulation trajectories.
A mean first-passage time (MFPT) is defined as the mean time needed for a particle to reach, starting from a given position $x_{s}$, a target position $x_{t}$ for the first time. ${ }^{32}$ In the main text, we calculate MFPTs from Langevin simulations via averaging the time a particle needs to go from one potential minimum $x_{s}=-L$ to the other potential minimum $x_{t}=L$. Each MFPT shown in the main text is obtained from one long trajectory and thus allows for multiple recrossing events. Our choice of $x_{t}$ makes sure that in the high-friction limit the particle recrosses the barrier with negligible probability right after reaching the target position and matches the above definition of the escape time.

We now compare escape times defined in Eqs. (A2) and (A5) with MFPTs, both calculated from simulations. We perform this comparison for simulations in the single-well as well as in the double-well scenarios. The double-well scenario, employed in the main text, consists of a single, long trajectory and allows for multiple recrossing events. For the single-well simulations, we use the same quartic potential Eq. (4) but impose an absorbing boundary at $x_{t}=L$, i.e., we stop the simulation once the particle reaches the minimum of the target well at $x_{t}=L$ for the first time. The single-well scenario thus consists of many simulation trajectories, each initialized as explained in the main text. The results, together with the predictions of both single- and double-well PGH theories, are shown in Fig. 7.

For the single-well simulation data, the escape time (squares) perfectly equals the MFPT (plus signs). This is easy to understand since both approaches impose an absorbing boundary at the target position $x_{t}=L$. For the double well, simulated escape times (circles) and MFPTs (crosses) only agree in the limit of high-friction and short memory time, for $\tau_{m} / \tau_{D} \ll 1$ and $\tau_{\Gamma} / \tau_{D} \ll 1$. In the energy diffusion regime, i.e., for large mass $\tau_{m} / \tau_{D} \gg 1$ or for long memory $\tau_{\Gamma} / \tau_{D}$ $\gg 1$, the simulated escape time is larger than the simulated MFPT by a factor of about 2 . The difference between escape
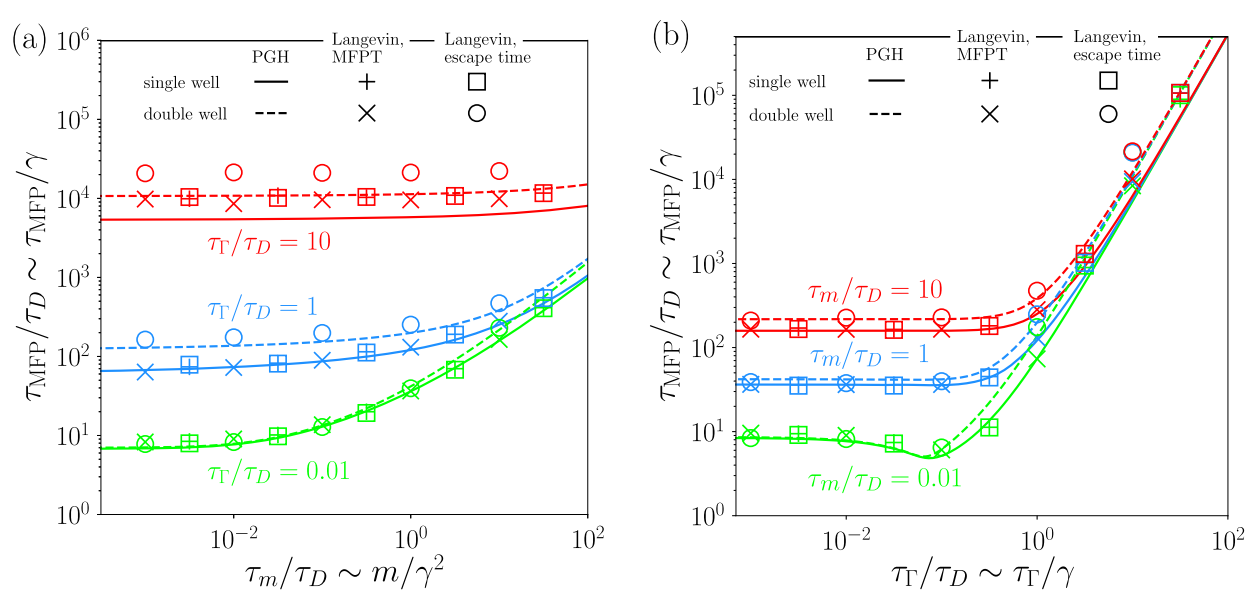

FIG. 7. Simulation results for $\tau_{\mathrm{MFP}}$ and $\tau_{\mathrm{esc}}$ in a single-well and double-well quartic potential together with predictions of the PGH theory, using the singlewell $^{41}$ (solid colored lines) and double-well ${ }^{36,47,48}$ (dashed colored lines) PGH formulas. The potential used for both single and double wells is given by Eq. (4), with a barrier height $U_{0}=3 k_{\mathrm{B}} T$. For the single well, an absorbing boundary is placed at $x_{t}=L$ so that only the region $x<L$ of the quartic potential is used. For the single well, MFPTs $\tau_{\text {MFP }}$ (plus signs) are calculated via direct sampling of first-passage times and escape times $\tau_{\text {esc }}$ (empty squares) are calculated

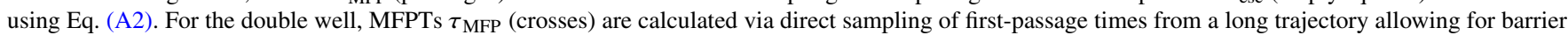
recrossing, as described in the main text and escape times $\tau_{\text {esc }}$ (empty circles) are calculated using Eq. (A5). (a) Plot of $\tau_{\text {MFP }} / \tau_{D}$ as a function of $\tau_{m} / \tau_{D}$ for several values of the rescaled memory time $\tau_{\Gamma} / \tau_{D}$ as denoted by the colored labels in the figure. (b) Plot of $\tau_{\mathrm{MFP}} / \tau_{D}$ as a function of $\tau_{\Gamma} / \tau_{D}$ for several values of $\tau_{m} / \tau_{D}$, as denoted by the colored labels in the figure. 
times and MFPTs has been discussed before ${ }^{32}$ and in our case has an intuitive explanation. In the energy diffusion regime, after the particle has crossed the barrier once, it has a high kinetic energy and will oscillate back and forth between the two wells multiple times, as is seen in the example trajectories in Figs. 1(b) and 1(f)-1(h). After the particle has stopped oscillating between the wells, it will be found in the target or in the starting well with equal probability. This fundamental difference between Markovian and non-Markovian barrier crossings is clearly seen in Figs. 1(d) and 1(h), where long trajectories in the high-friction Markovian regime (exhibiting no recrossings) and in the high-friction non-Markovian regime (with multiple recrossings) are shown. Thus, in the energy diffusion regime, encompassing both the inertial case and also the high-friction non-Markovian case, only half of the barrier-crossing events will be productive and bring the particle from the starting to the target well. This explains why the escape time becomes twice the MFPT in the energy diffusion regime, namely, because in the calculation of a MFPT, each barrier crossing event is counted, regardless of whether it is productive or not.

On the other hand, the simulated single-well and doublewell MFPTs agree well with each other and also with the single-well escape times for all parameters considered, thus only the escape time for the double-well system yields different results from the other scenarios and becomes twice as large as the other barrier crossing times in the energy diffusion regime for $\tau_{m} / \tau_{D} \gg 1$ or $\tau_{\Gamma} / \tau_{D} \gg 1$. From the fact that double-well and single-well MFPTs agree with each other, we conclude that recrossing events, which are definitely present in the calculation of MFPTs in a double well, contribute negligibly to the statistical average in $\tau_{\text {MFP. This is a nontrivial }}$ and noteworthy finding.

The difference between escape times in the single-well and double-well scenarios in the energy diffusion regime is also obtained in PGH theories, shown as solid and dashed lines in Fig. 7. While in the regime of high friction and short memory, both lines agree, in the regime of long memory or large mass, the two lines differ by a factor of about 2, similar to our simulation results. Overall, the PGH theory matches the numerical escape times remarkably well for both the singlewell and the double-well scenarios. Only for long memory $\tau_{\Gamma} / \tau_{D} \gg 1$, the PGH predictions are smaller than the numerical results by a factor of about 2 , as can most clearly be seen in Fig. 7(a) by comparing the numerical escape times for $\tau_{\Gamma} / \tau_{D}=10$ to the corresponding PGH theory predictions. Note that the small deviation between PGH predictions and simulated escape times happens to be of the same order of magnitude as the difference between the simulated MFPTs and escape times in the double-well scenario, which we believe to be purely coincidental. The difference between simulation and PGH predictions for the escape times could be caused by imperfections of the PGH theory but also by numerical inaccuracies.

In conclusion, the MFPTs we calculate in the main text from simulations in a double well can rightfully be compared to escape times calculated within the single-well PGH theory. The reason is that MFPTs within the double-well scenario (which is numerically the most efficient scenario to obtain barrier crossing times) agree perfectly with escape times (or MFPTs) obtained from the single-well scenario, as demonstrated by our simulations in Fig. 7.

\section{APPENDIX B : COMPARISON OF QUARTIC AND CUBIC POTENTIALS}

Based on the PGH expression for the escape time from a quartic single well, we construct the heuristic formula Eq. (9), which describes MFPTs calculated from Langevin simulations in a quartic potential very well. In this appendix, we show that the heuristic formula Eq. (9) also captures the MFPTs in a cubic potential. To allow for a meaningful comparison, we consider a cubic single-well potential,

$$
U(x)=-2 U_{0} \frac{x}{L}\left[\left(\frac{x}{L}+\frac{3}{4}\right)^{2}-\frac{9}{16}\right]+U_{0},
$$
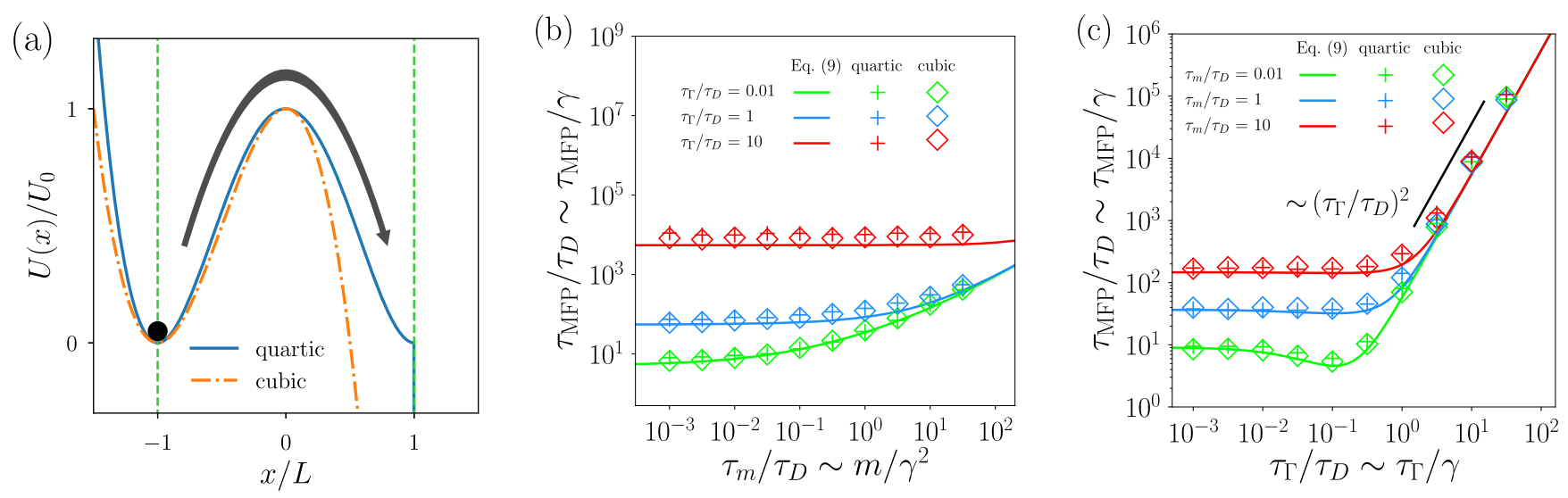

FIG. 8. (a) Illustration of the barrier crossing of a particle in the potentials $U(x)$ defined in Eqs. (B1) and (B2). The mean first-passage time $\tau_{\text {MFP is defined as }}$ the mean time difference between crossing the minimum at the starting position $x_{s}=-L$ (left vertical dashed line) and reaching the target position $x_{t}=L$ (right vertical dashed line) for the first time. [(b) and (c)] MFPTs obtained from Langevin simulations using the potentials shown in subplot (a) are compared to the heuristic formula Eq. (9). For each datapoint, thousands of simulations are run for $U_{0}=3 k_{\mathrm{B}} T$ with stochastic initial conditions as described in the main text and using an absorbing boundary condition at $x_{t}=L$. While subplot (b) shows MFPTs as a function of $\tau_{m} / \tau_{D}$ for various values of $\tau_{\Gamma} / \tau_{D}$, subplot (c) shows MFPTs as a function of $\tau_{\Gamma} / \tau_{D}$ for various values of $\tau_{m} / \tau_{D}$. 

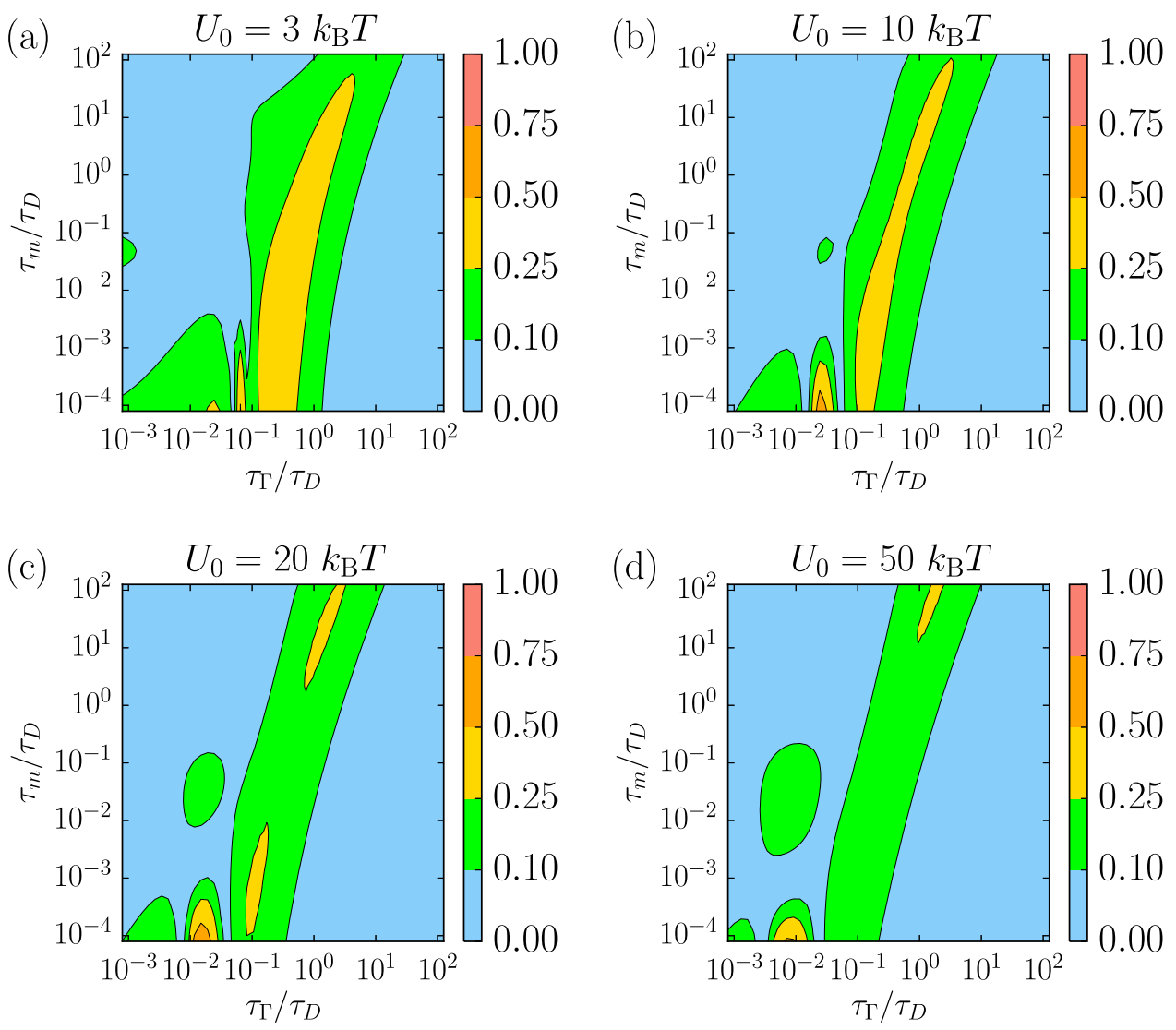

FIG. 9. The subplots show the relative deviation, as defined by Eq. (C1), between the PGH theory and the heuristic formula, Eq. (9), for barrier heights $U_{0}=3,10,20$, and $50 k_{\mathrm{B}} T$. and a quartic single-well potential,

$$
U(x)= \begin{cases}U_{0}\left[\left(\frac{x}{L}\right)^{2}-1\right]^{2} & x \leq L, \\ -\infty & x>L .\end{cases}
$$

The two potentials are shown in Fig. 8(a), both have a barrier height $U_{0}$ and a distance $L$ between the potential minimum at $x=-L$ and the barrier top. For each potential, we run many simulations using initial conditions as explained in the main text and absorbing boundary conditions at the target position $x_{t}=L$ so that a simulation is stopped once the particle reaches $x_{t}$ for the first time. Resulting MFPTs are shown together with the heuristic formula Eq. (9) in Figs. 8(b) and 8(c). We find that the two potentials (B1) and (B2) lead to almost identical MFPTs, which are well described by the heuristic formula Eq. (9). Only in the long memory time regime $\tau_{\Gamma} / \tau_{D} \gtrsim 1$, the heuristic formula systematically yields MFPTs that are slightly smaller than the Langevin results for both potentials. These small discrepancies are expected since the PGH theory shows similar behavior, see Fig. 2, and since the heuristic formula is based on the PGH theory.

We conclude that our heuristic formula Eq. (9), using as only input the barrier height $U_{0}$ and the distance $L$ between the minimum at $x=-L$ and the barrier maximum at $x=0$, accurately predicts MFPTs for different potential shapes and is not limited to the quartic double well Eq. (4) we consider in the main text. This is expected to hold as long as the curvatures at the potential minimum and the barrier top are not very different. If in contrast one has a narrow well and a broad barrier top or vice versa, the predictions of the PGH theory can be very different from the rather symmetric scenario we use to construct our heuristic formula ${ }^{31}$ so that the full PGH theory should be employed in such situations.

\section{APPENDIX C : RELATIVE DEVIATION BETWEEN HEURISTIC FORMULA AND PGH THEORY}

To demonstrate the global validity of Eq. (9), we show in Fig. 9 contour plots of the relative deviation,

$$
\frac{\Delta \tau_{\mathrm{MFP}}}{\tau_{\mathrm{MFP}}} \equiv\left|\frac{\tau_{\mathrm{MFP}}-\tau_{\mathrm{MFP}}^{\mathrm{PGH}}}{\tau_{\mathrm{MFP}}^{\mathrm{PGH}}}\right|,
$$

between the MFPT as predicted by the PGH theory, denoted here by $\tau_{\mathrm{MFP}}^{\mathrm{PGH}}$, and the heuristic formula (9), denoted here by

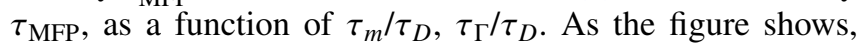
the relative deviation between the heuristic formula and PGH theory, whose predictions for $\tau_{\text {MFP }}$ range over 27 orders of magnitude in the parameter range considered, is typically of the order of $10 \%$.

\footnotetext{
${ }^{1}$ A. Berezhkovskii and A. Szabo, J. Chem. Phys. 122, 014503 (2005).

${ }^{2}$ J. D. Bryngelson, J. N. Onuchic, N. D. Socci, and P. G. Wolynes, Proteins: Struct., Funct., Genet. 21, 167 (1995).

${ }^{3}$ J. D. Bryngelson and P. G. Wolynes, J. Phys. Chem. 93, 6902 (1989).

${ }^{4}$ O. Dudko, G. Hummer, and A. Szabo, Phys. Rev. Lett. 96, 108101 (2006).

${ }^{5}$ N. D. Socci, J. N. Onuchic, and P. G. Wolynes, J. Chem. Phys. 104, 5860 (1996).

${ }^{6}$ H. Orland, J. Chem. Phys. 134, 174114 (2011).

${ }^{7}$ H. S. Chung, S. Piana-Agostinetti, D. E. Shaw, and W. A. Eaton, Science 349, 1504 (2015).

${ }^{8}$ K. Truex, H. S. Chung, J. M. Louis, and W. A. Eaton, Phys. Rev. Lett. 115, 018101 (2015).

${ }^{9}$ K. Neupane, A. P. Manuel, and M. T. Woodside, Nat. Phys. 12, 700 (2016).

${ }^{10}$ R. Best and G. Hummer, Phys. Rev. Lett. 96, 228104 (2006).

${ }^{11}$ W. Zheng and R. B. Best, J. Phys. Chem. B 119, 15247 (2015).
} 
${ }^{12}$ J. Ashcroft, M. Besnard, V. Aquada, and J. Jonas, Chem. Phys. Lett. 110, 420 (1984).

${ }^{13}$ R. B. Best and G. Hummer, Proc. Natl. Acad. Sci. U. S. A. 102, 6732 (2005).

${ }^{14}$ O. K. Dudko, T. G. W. Graham, and R. B. Best, Phys. Rev. Lett. 107, 208301 (2011).

${ }^{15}$ B. Peters and B. L. Trout, J. Chem. Phys. 125, 054108 (2006).

${ }^{16}$ M. A. Rohrdanz, W. Zheng, M. Maggioni, and C. Clementi, J. Chem. Phys. 134, 124116 (2011).

${ }^{17}$ A. V. Mokshin, R. M. Yulmetyev, and P. Hänggi, Phys. Rev. Lett. 95, 200601 (2005).

${ }^{18}$ G. Hummer, J. Chem. Phys. 120, 516 (2004).

${ }^{19}$ B. Peters, Annu. Rev. Phys. Chem. 67, 669 (2016)

${ }^{20}$ R. G. Mullen, J.-E. Shea, and B. Peters, J. Chem. Theory Comput. 10, 659 (2014).

${ }^{21}$ P. G. Bolhuis, D. Chandler, C. Dellago, and P. L. Geissler, Annu. Rev. Phys. Chem. 53, 291 (2002).

${ }^{22}$ D. de Sancho, A. Sirur, and R. B. Best, Nat. Commun. 5, 4307 (2014).

${ }^{23}$ O. F. Lange and H. Grubmüller, J. Chem. Phys. 124, 214903 (2006).

${ }^{24}$ R. O. Rosenberg, B. J. Berne, and D. Chandler, Chem. Phys. Lett. 75, 162 (1980).

${ }^{25}$ J. M. Sancho, A. H. Romero, and K. Lindenberg, J. Chem. Phys. 109, 9888 (1998).

${ }^{26}$ D. M. Zuckerman and T. B. Woolf, J. Chem. Phys. 116, 2586 (2002).

${ }^{27}$ Z. Li, X. Bian, X. Li, and G. E. Karniadakis, J. Chem. Phys. 143, 243128 (2015).

${ }^{28}$ R. Rey and E. Guardia, J. Phys. Chem. 96, 4712 (1992).

${ }^{29}$ R. Zwanzig, Phys. Rev. 124, 983 (1961).

${ }^{30}$ H. Mori, Prog. Theor. Phys. 33, 423 (1965).
${ }^{31}$ J. E. Straub, M. Borkovec, and B. J. Berne, J. Chem. Phys. 84, 1788 (1986).

${ }^{32}$ P. Reimann, G. J. Schmid, and P. Hänggi, Phys. Rev. E 60, R1 (1999).

${ }^{33}$ S. Arrhenius, Z. Phys. Chem. 4, 226 (1889).

${ }^{34}$ H. Eyring, J. Chem. Phys. 3, 107 (1935).

${ }^{35}$ H. Kramers, Physica 7, 284 (1940).

${ }^{36}$ V. I. Mel'nikov and S. V. Meshkov, J. Chem. Phys. 85, 1018 (1986).

${ }^{37}$ R. F. Grote and J. T. Hynes, J. Chem. Phys. 73, 2715 (1980).

${ }^{38}$ B. Carmeli and A. Nitzan, Phys. Rev. Lett. 49, 423 (1982).

${ }^{39}$ P. Hänggi, J. Stat. Phys. 42, 105 (1986).

${ }^{40}$ P. Talkner and H.-B. Braun, J. Chem. Phys. 88, 7537 (1988).

${ }^{41}$ E. Pollak, H. Grabert, and P. Hänggi, J. Chem. Phys. 91, 4073 (1989).

${ }^{42}$ R. Zwanzig, J. Stat. Phys. 9, 215 (1973).

${ }^{43}$ E. Pollak and R. Ianconescu, J. Chem. Phys. 140, 154108 (2014).

${ }^{44}$ E. Pollak and R. Ianconescu, J. Phys. Chem. A 120, 3155 (2016).

${ }^{45}$ R. Ianconescu and E. Pollak, J. Chem. Phys. 143, 104104 (2015).

${ }^{46}$ P. Hänggi, P. Talkner, and M. Borkovec, Rev. Mod. Phys. 62, 251 (1990).

${ }^{47}$ V. Mel'nikov, Phys. Rep. 209, 1 (1991).

${ }^{48}$ E. Pollak, in Theoretical Methods in Condensed Phase Chemistry, edited by S. D. Schwartz (Kluwer Academic Publishers, Dordrecht, 2002), Vol. 5, pp. 1-46.

${ }^{49}$ D. A. Rose and I. Benjamin, J. Chem. Phys. 100, 3545 (1994).

${ }^{50}$ D. E. Smith and L. X. Dang, J. Chem. Phys. 100, 3757 (1994).

${ }^{51}$ M. Roca, V. Moliner, I. Tuñón, and J. T. Hynes, J. Am. Chem. Soc. 128, 6186 (2006).

${ }^{52}$ L. X. Dang and G. K. Schenter, Chem. Phys. Lett. 643, 142 (2016).

${ }^{53}$ H. V. R. Annapureddy and L. X. Dang, J. Phys. Chem. B 118, 8917 (2014).

${ }^{54}$ B. Carmeli and A. Nitzan, Phys. Rev. Lett. 51, 233 (1983).

${ }^{55}$ D. Chandler, J. Chem. Phys. 68, 2959 (1978). 